\title{
Vital Signs: Surveillance for Acute Flaccid Myelitis — United States, 2018
}

\author{
Adriana Lopez, MHS ${ }^{1}$; Adria Lee, $\mathrm{MPH}^{1}$; Angela Guo, MPH${ }^{1}$; Jennifer L. Konopka-Anstadt, PhD ${ }^{1}$; Amie Nisler, MPH ${ }^{1}$; Shannon L. Rogers, MS ${ }^{1}$; \\ Brian Emery ${ }^{1}$; W. Allan Nix ${ }^{1}$; Steven Oberste, $\mathrm{PhD}^{1}$; Janell Routh, $\mathrm{MD}^{1}$; Manisha Patel, $\mathrm{MD}^{1}$
}

On July 9, 2019, this report was posted as an MMWR Early Release on the MMWR website (https://www.cdc.gov/mmwr).

\begin{abstract}
Background: Acute flaccid myelitis (AFM), a serious paralytic illness, was first recognized as a distinct condition in 2014, when cases were reported concurrent with a large U.S. outbreak of severe respiratory illness caused by enterovirus D-68 (EV-D68). Since 2014, nationwide outbreaks of AFM have occurred every 2 years in the United States; the cause for the recent change in the epidemiology of AFM in the United States, including the occurrence of outbreaks and a biennial periodicity since 2014, is under investigation. This report updates clinical, laboratory, and outcome data for cases reported to CDC during 2018.

Methods: Clinical data and specimens from persons in the United States who met the clinical criterion for AFM (acute onset of flaccid limb weakness) with onset in 2018 were submitted to CDC for classification of the illnesses as confirmed, probable, or non-AFM cases. Enterovirus/rhinovirus (EV/RV) testing was performed on available specimens from persons meeting the clinical criterion. Descriptive analyses, laboratory results, and indicators of early recognition and reporting are summarized.

Results: From January through December 2018, among 374 reported cases of AFM, 233 (62\%) (from 41 states) were classified as confirmed, $26(7 \%)$ as probable, and $115(31 \%)$ as non-AFM cases. Median ages of patients with confirmed, probable, and non-AFM cases were 5.3, 2.9, and 8.8 years, respectively. Laboratory testing identified multiple EV/RV types, primarily in respiratory and stool specimens, in $44 \%$ of confirmed cases. Among confirmed cases, the interval from onset of limb weakness until specimen collection ranged from 2 to 7 days, depending on specimen type. Interval from onset of limb weakness until reporting to CDC during 2018 ranged from 18 to 36 days, with confirmed and probable cases reported earlier than non-AFM cases.

Conclusion: Identification of risk factors leading to outbreaks of AFM remains a public health priority. Prompt recognition of signs and symptoms, early specimen collection, and complete and rapid reporting will expedite public health investigations and research studies to elucidate the recent epidemiology of AFM and subsequently inform treatment and prevention recommendations.
\end{abstract}

\section{Introduction}

Acute flaccid myelitis (AFM) was initially defined as a distinct entity in 2014 following reports of the occurrence of acute limb weakness in previously healthy children across the United States during an outbreak of severe respiratory disease caused by enterovirus D-68 (EV-D68) $(1,2)$. AFM is a rare but serious illness for which there are no known treatments or means of prevention. It is a recognized complication of infections caused by West Nile Virus, adenovirus, and enteroviruses $(3,4)$; however, the more recent epidemiology of AFM, including the occurrence of outbreaks, its biennial periodicity since 2014, and the clustering of cases during the late summer and fall, has not been previously observed.

Neuroinvasive enteroviruses have been identified as causes of sporadic cases of AFM, including EV-D68 and EV-A71
(5-8). Extensive testing of AFM cases confirmed as part of national surveillance has detected multiple enteroviruses from sterile sites (i.e., cerebrospinal fluid [CSF] and serum) and nonsterile specimens (i.e., respiratory specimens and stool). Lack of a clear explanation for the emergence, in addition to the overall rarity of this condition, pose substantial challenges for identifying optimal treatment and prevention measures. Increased awareness of AFM by clinicians and timely reporting of persons with symptoms consistent with AFM to public health authorities are essential to identifying cases, improving patient management, and initiating public health investigations to further understand this condition.

This report summarizes and updates surveillance data for suspected cases of AFM reported to CDC (9), with onset of 


\section{Summary}

What is already known about this topic?

Biennial U.S. outbreaks of acute flaccid myelitis (AFM) have been recognized since 2014. Most cases occur in children during late summer and early fall.

What is added by this report?

During 2018, 233 confirmed AFM cases were reported, the largest number since surveillance began in 2014. Upper limb involvement only was more prevalent in confirmed cases (42\%), as was report of respiratory symptoms or fever (92\%) within 4 weeks preceding limb weakness onset. Median intervals from onset of limb weakness to hospitalization, magnetic resonance imaging, and reporting to CDC were 1,2 , and 18 days, respectively.

What are the implications for public health practice?

Prompt recognition, early specimen collection, and rapid reporting will expedite public health investigations and help characterize AFM.

flaccid limb weakness from January 1 through December 31, 2018. Data from 2018 were also compared with the previous peak of AFM in 2016 to identify opportunities to improve recognition and reporting.

\section{Methods}

Health departments submitted reports of patients meeting the clinical criterion for AFM (acute onset of flaccid limb weakness) to CDC for case classification. For public health surveillance purposes, a confirmed case of AFM was defined as acute flaccid limb weakness in a person with magnetic resonance imaging (MRI) evidence of a spinal cord lesion largely restricted to gray matter and spanning $\geq 1$ spinal segments. Patients with probable AFM met the clinical criterion and had CSF pleocytosis ( $>5$ white blood cells per cubic mm) (10). Patients without documented flaccid limb weakness, with MRI findings that were inconsistent with AFM, or who had alternative diagnoses (e.g., transverse myelitis, acute disseminated encephalomyelitis, Guillain-Barré syndrome, other myelopathy, or spinal stroke) were classified as non-AFM cases.

Health departments and clinicians submitted CSF, respiratory, serum, and/or stool specimens, when available, from patients with suspected AFM to CDC for testing (https:// www.cdc.gov/acute-flaccid-myelitis/hcp/instructions.html). In accordance with current clinical, laboratory, and epidemiologic evidence, CDC laboratory protocols included testing of these specimens for enteroviruses, rhinoviruses, and parechoviruses. All specimens were tested for EV/RV using a 5' nontranslated region qualitative real-time reverse transcription-polymerase chain reaction (real-time RT-PCR) pan-enterovirus assay (11) and a pan-enterovirus typing assay by viral protein 1 RT-semi-nested PCR and Sanger sequencing of the resultant amplicon (12). All specimens were also tested for parechoviruses using a pan-parechovirus real-time RT-PCR assay (13). Stool specimens were tested for poliovirus by virus isolation in cell culture as part of national poliovirus surveillance. A subset of 31 specimens was also tested at CDC for arboviruses. Results from non-CDC laboratories are not included in this update.

Descriptive analyses of confirmed, probable, and non-AFM cases in patients with onset of limb weakness in 2018 were performed using SAS (version 9.4; SAS Institute). To ascertain early recognition of AFM by clinicians, the number of days from onset of limb weakness to hospitalization and receipt of MRI were compiled. Data from cases confirmed in 2016 and 2018 were compared to evaluate time to hospitalization, collection of specimens, and reporting to CDC. Categorical variables were compared using Chi-squared tests, and medians were compared using the Wilcoxon rank sum test. P-values of $<0.05$ were considered statistically significant.

\section{Results}

Since surveillance for AFM began following the 2014 outbreak, nationwide outbreaks have occurred in 2016 and 2018 (Figure 1). From January 1 through December 31, 2018, a total of 374 persons meeting the clinical criterion for AFM were reported to CDC; 233 (62\%), from 41 states, were classified as confirmed, $26(7 \%)$ as probable, and $115(31 \%)$ as non-AFM cases (Figure 2). The median age of patients with confirmed AFM, 5.3 years (range $=6$ months -81.8 years), was significantly older than that of patients with probable AFM (2.9 years $[$ range $=4$ months -55.3 years] $; \mathrm{p}=0.04$ ). Patients with illnesses classified as non-AFM were significantly older than were patients with confirmed AFM (median $=8.6$ years [range $=1$ month-78.1 years]; $\mathrm{p}<0.001)$ (Table 1$)$. Sex and race did not differ among patients with confirmed AFM, probable AFM, and non-AFM. Involvement of upper limbs only was significantly more prevalent in patients with confirmed AFM $(42 \%)$ than in those with non-AFM (10\%) ( $<<0.001)$. Patients with confirmed and probable AFM more frequently had respiratory symptoms (e.g., cough, rhinorrhea, and congestion) or fever (e.g., temperature $\left.\geq 100.4^{\circ} \mathrm{F}\right)(92 \%)$ within the 4 weeks preceding limb weakness onset than did patients with non-AFM (62\%; $\mathrm{p}<0.001)$. Among all patients with confirmed, probable, and non-AFM, 227 (98\%), 26 (100\%), and 113(98\%), respectively, were hospitalized, including 127 (60\%), 12 (57\%), and 54 (50\%), respectively, admitted to an intensive care unit; $27 \%$ (62) of those with confirmed AFM required respiratory support, $87 \%$ of whom (53/61) required mechanical ventilation. No deaths were reported during the acute illness of patients with confirmed AFM who had limb weakness onset in 2018; however, there were two reports of patients confirmed with AFM in 2018 who had died months after limb weakness onset. 
FIGURE 1. Confirmed cases of acute flaccid myelitis reported to CDC ( $N=559)$ - United States, August 1, 2014-December 31, 2018

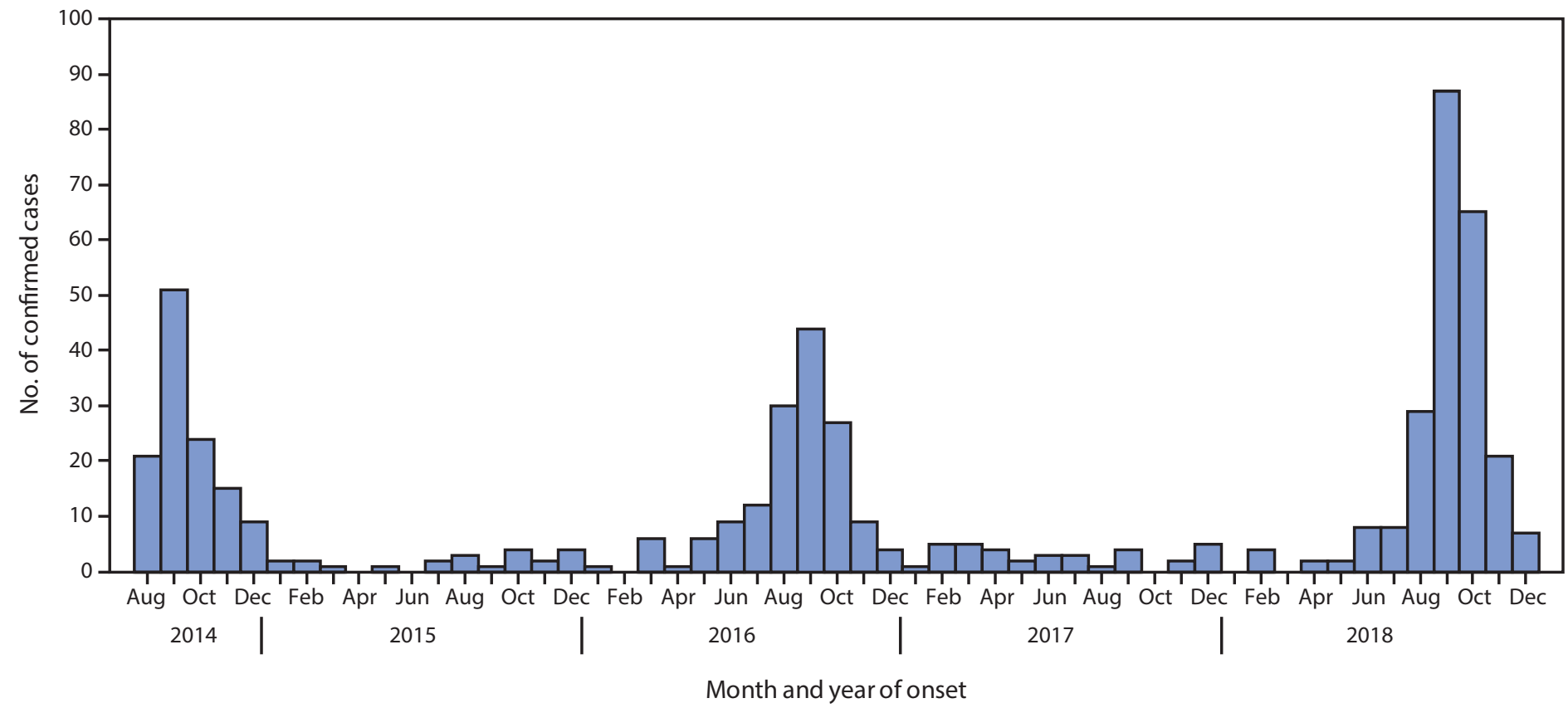

FIGURE 2. Cases of acute flaccid myelitis reported to CDC, by case classification status — United States, 2018

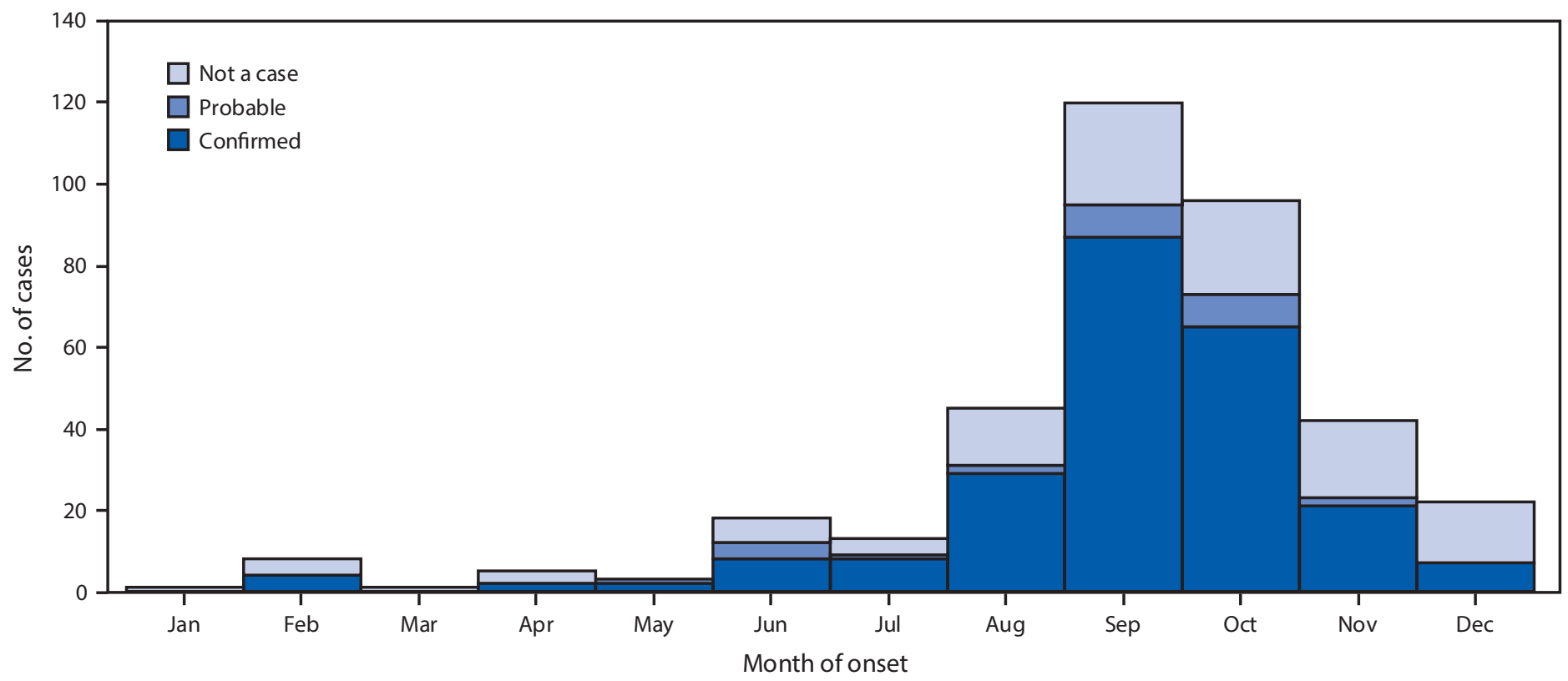

Among patients with confirmed AFM, the interval between limb weakness onset to hospitalization (1 day) and to MRI (2 days) suggests early recognition by clinicians. Among patients with probable AFM, the interval from onset of limb weakness to hospitalization (3 days) and MRI (4 days) was significantly longer than that among those with confirmed AFM. Compared with patients with confirmed AFM, the interval from onset of limb weakness to hospitalization among patients with non-AFM (1 day) was similar, but the interval to MRI (3 days) was significantly longer $(\mathrm{p}=0.002)$. Among patients with confirmed AFM, the median interval from onset of limb weakness to specimen collection ranged from 2-7 days, depending on specimen type. The median interval from onset of limb weakness until reporting to CDC ranged from 18-36 days, with confirmed and probable cases reported earlier than non-AFM cases (Table 1). 
TABLE 1. Demographic and clinical characteristics of patients with confirmed and probable cases of acute flaccid myelitis (AFM) and non-AFM cases, and timing to medical care and reporting to public health — United States, 2018

\begin{tabular}{|c|c|c|c|c|c|}
\hline \multirow[b]{2}{*}{ Characteristic } & \multicolumn{2}{|c|}{ No. (\%) } & \multirow[b]{2}{*}{ P-value* } & \multirow{2}{*}{$\frac{\text { No. }(\%)}{\text { Noncase }(\mathrm{N}=115)}$} & \multirow[b]{2}{*}{ P-value ${ }^{\dagger}$} \\
\hline & Confirmed (N = 233) & Probable $(\mathrm{N}=26)$ & & & \\
\hline \multicolumn{6}{|l|}{ Demographics } \\
\hline Median age, yrs (range, IQR) & $5.3(0.5-81.8,3.3-8.2)$ & $2.9(0.3-55.3,1.0-10.1)$ & 0.04 & $8.8(0.1-78.1,3.9-19.7)$ & $<0.001$ \\
\hline Male sex & $136 / 233(58)$ & $14 / 25(56)$ & 0.83 & $67 / 111(60)$ & 0.81 \\
\hline \multicolumn{6}{|l|}{ Race } \\
\hline Asian & 9/233 (4) & $1 / 26(4)$ & 0.87 & 8/115 (7) & 0.40 \\
\hline Black or African American & $22 / 233(9)$ & $4 / 26(15)$ & & $17 / 115(15)$ & \\
\hline Native Hawaiian/Pacific Islander & $1 / 233(0)$ & $0 / 26(0)$ & & $0 / 115(0)$ & \\
\hline White & $147 / 233(63)$ & $14 / 26(54)$ & & $69 / 115(60)$ & \\
\hline Multiracial & $4 / 233(2)$ & $1 / 26(4)$ & & $1 / 115(1)$ & \\
\hline Unknown & $50 / 233(21)$ & $6 / 26(23)$ & & $20 / 115(17)$ & \\
\hline \multicolumn{6}{|l|}{ Laboratory finding } \\
\hline Lumbar puncture & 219/229 (96) & $26 / 26(100)$ & 0.60 & 102/111 (92) & 0.21 \\
\hline Pleocytosis & 180/207 (87) & $26 / 26(100)$ & 0.05 & $46 / 88(52)$ & $<0.001$ \\
\hline Median ${ }^{\S}$, cells $/ \mathrm{mm}^{3}$ (range, IQR) & $92(6-814,42-158)$ & $42(7-730,16-128)$ & 0.01 & $53(7-920,27-146)$ & 0.19 \\
\hline Spine MRI performed & $231 / 232(99)$ & $25 / 26(96)$ & 0.19 & $109 / 114(96)$ & 0.02 \\
\hline \multicolumn{6}{|l|}{ Clinical illness } \\
\hline Upper limbs only & $98 / 233(42)$ & $6 / 26(23)$ & 0.09 & $12 / 115(10)$ & $<0.001$ \\
\hline Lower limbs only & $31 / 233(13)$ & $8 / 26(31)$ & 0.04 & $30 / 115(26)$ & 0.004 \\
\hline \multicolumn{6}{|c|}{ In the 4 weeks before onset of limb weakness } \\
\hline Any illness & $219 / 229(96)$ & $25 / 26(96)$ & 1.00 & $85 / 108(79)$ & $<0.001$ \\
\hline Any respiratory illness & $184 / 222(83)$ & $18 / 26(69)$ & 0.11 & $54 / 109(50)$ & $<0.001$ \\
\hline Any fever & $170 / 217(78)$ & $19 / 24(79)$ & 1.00 & $46 / 101(46)$ & $<0.001$ \\
\hline Any respiratory illness or fever & $214 / 233(92)$ & $24 / 26(92)$ & 1.00 & $71 / 115(62)$ & $<0.001$ \\
\hline Any gastrointestinal illness & $80 / 225(36)$ & $9 / 26(35)$ & 1.00 & $42 / 108(39)$ & 0.63 \\
\hline Hospitalized & $227 / 231(98)$ & $26 / 26(100)$ & 1.00 & $113 / 115(98)$ & 1.00 \\
\hline ICU & $127 / 210(60)$ & $12 / 21(57)$ & 0.82 & $54 / 107(50)$ & 0.09 \\
\hline \multicolumn{6}{|c|}{ Timing of preceding illness to onset of limb weakness, median days (range, IQR) } \\
\hline Any illness & $5(0-27,2-8)$ & $4(0-19,2-10)$ & 0.84 & $5(0-28,2-10)$ & 0.78 \\
\hline Any respiratory illness & $5(0-27,3-8)$ & $4(0-19,3-11)$ & 0.67 & $6.5(0-28,3-11.5)$ & 0.63 \\
\hline Any fever & $3(0-21,1-5)$ & $3(0-19,1.5-8.5)$ & 0.25 & $4(0-28,1-7)$ & 0.12 \\
\hline Any respiratory illness or fever & $5(0-27,2-7)$ & $3(0-19,2-11)$ & 0.77 & $5(0-28,2-10)$ & 0.40 \\
\hline Any gastrointestinal illness & $2.5(0-23,1-7)$ & $4(0-17,2-5)$ & 0.61 & $4(0-19,1-6.5)$ & 0.22 \\
\hline \multicolumn{6}{|c|}{ Timing from onset of limb weakness to medical care, specimen collection, and reporting to public health, median days (range, IQR) } \\
\hline Hospitalization & $1(0-54,0-2)$ & $3(0-8,1-4)$ & 0.03 & $1(0-62,0-3)$ & 0.48 \\
\hline Lumbar puncture & $2(0-31,1-3)$ & $4(0-30,1-7)$ & 0.03 & $2(0-140,1-5)$ & 0.05 \\
\hline MRI & $2(0-164,1-3)$ & $4(0-12,2-7)$ & 0.02 & $3(0-113,1-8)$ & 0.002 \\
\hline \multicolumn{6}{|l|}{ Specimen collection } \\
\hline CSF & $2(0-31,1-4)$ & $7(2-19,6-11)$ & 0.01 & $5(0-63,2-9)$ & 0.09 \\
\hline Respiratory & $3(0-35,2-6)$ & $13(2-65,6-21)$ & 0.004 & $6(1-66,3-11)$ & 0.03 \\
\hline Serum & $4(0-31,2-7)$ & $9(3-65,6-19)$ & 0.007 & $8.5(1-64,5-14)$ & $<0.001$ \\
\hline Stool & $7(0-44,4-11)$ & $13(2-65,6-17)$ & 0.13 & $8(0-65,6-14)$ & 0.33 \\
\hline Completion of patient summary form & $8.5(1-175,4-25)$ & $14(4-105,8-21)$ & 0.10 & $20(0-277,9-56)$ & $<0.001$ \\
\hline CDC notified & $18(0-208,7-35)$ & $18.5(4-111,12-26)$ & 0.75 & $36(1-282,14-70)$ & 0.003 \\
\hline
\end{tabular}

Abbreviations: $\mathrm{CSF}=$ cerebrospinal fluid; $\mathrm{ICU}=$ intensive care unit; $\mathrm{IQR}=$ interquartile range; $\mathrm{MRI}=$ magnetic resonance imaging

* P-value represents comparison of confirmed and probable cases of AFM.

† P-value represents comparison of confirmed and non-AFM cases.

$\S$ Median includes only those cases with pleocytosis ( $>5$ white blood cells per cubic $\mathrm{mm}$ ).

Among all 233 patients with confirmed AFM, CSF, respiratory specimens, and stool specimens were tested from $74(32 \%)$, 123 (53\%), and 100 (43\%) patients, respectively (Table 2). The highest positive yield (44\%) was from respiratory specimens, of which EV-D68 was most commonly detected; only two (3\%) CSF specimens tested positive (one each for EV-D68 and EV-A71). Testing of specimens from probable and non-AFM cases also identified multiple EV/RV types. Stool specimens from all patients with available specimens tested negative for poliovirus.
Among specimens sent from 31 patients (17 confirmed, three probable, and 11 noncases) for arboviral testing, all were negative.

Among patients with confirmed AFM in 2018, the median interval between antecedent illness (e.g., febrile, respiratory, and/or gastrointestinal) during the preceding 4 weeks and onset of limb weakness ( 5 days), between limb weakness and hospitalization ( 1 day) and CSF collection (2 days) was similar to that in the 2016 outbreak, ( 5 days, 1 day, and 3 days, respectively) (Supplementary table, https://stacks.cdc.gov/view/cdc/79271). 
TABLE 2. Laboratory results from cerebrospinal fluid (CSF), respiratory, and stool specimens collected from patients with confirmed acute flaccid myelitis ( $\mathrm{N}=233$ ) - United States, 2018

\begin{tabular}{|c|c|c|c|}
\hline Specimen source & $\begin{array}{l}\text { No. with specimens available } \\
\text { (\% of } 233)\end{array}$ & No. (\%) positive & Positive test results (No.) \\
\hline CSF & $74(32)$ & $2 / 74(3)$ & $\begin{array}{l}\text { EV-A71 (1) } \\
\text { EV-D68 (1) }\end{array}$ \\
\hline Respiratory & $123(53)$ & $54 / 123(44)$ & $\begin{array}{c}\text { EV-D68 (30) } \\
\text { EV-A71 (10) } \\
\text { Other/Untyped EV/RV (14) }\end{array}$ \\
\hline Stool & $100(43)$ & $13 / 100(13)$ & $\begin{array}{c}\text { EV-D68 (1) } \\
\text { EV-A71 (2) } \\
\text { Echovirus 11 (1) } \\
\text { Coxsackievirus (3) } \\
\text { Parechovirus (4) } \\
\text { Other/Untyped EV/RV (2) }\end{array}$ \\
\hline
\end{tabular}

Abbreviations: $\mathrm{EV}=$ enterovirus; $\mathrm{RV}=$ rhinovirus.

However, the median interval from onset of limb weakness to MRI, respiratory specimen collection, and stool collection was shorter in 2018 than in 2016 ( 2 days versus 3 days, 3 days versus 4.5 days, and 7 days versus 7.5 days, respectively). Reporting to CDC occurred at a median of 18 days (range $=0-208$ days) in 2018 versus 15 days (range $=0-344$ days) in 2016 for patients with confirmed AFM.

\section{Discussion}

National AFM surveillance using a standardized case definition was established following the first recognized outbreak in 2014. Subsequently, two nationwide outbreaks occurred, one in 2016 with 149 confirmed cases (https://www.cdc.gov/acuteflaccid-myelitis/afm-surveillance.html), ${ }^{*}$ and the largest in 2018, which accounted for $42 \%$ of the 559 cases reported from August 2014 through December 2018. As in previous years, most AFM cases occurred in children, during the late summer and early fall. Findings such as presence of fever or respiratory symptoms before the onset of limb weakness, predominance of upper limb involvement, and detection of viruses in respiratory specimens in approximately $50 \%$ of patients with specimens submitted were also consistent with those in previous outbreak years. The accumulation of national surveillance data since 2014 has been pivotal to refining the AFM case definition, allowing for better differentiation of epidemiologic, clinical, and laboratory features and risk factors of confirmed cases from those of probable and non-AFM cases. In addition, the shorter interval between limb weakness and diagnostic evaluations in 2018 compared with that in 2016, suggests that support to health departments for strengthened surveillance and increased provider outreach activities has improved awareness of AFM among providers, particularly during outbreak years. Prompt recognition, early specimen

\footnotetext{
* Numbers for 2018 include patients under investigation that have not yet been classified.
}

collection, and reporting of all suspected cases to public health are important goals for AFM national surveillance.

Early recognition and specimen collection from suspected AFM patients are essential to optimizing pathogen detection and determining whether single or multiple etiologies are responsible for the recent outbreaks. Data from the 2014 and 2016 outbreaks suggested that early specimen collection resulted in higher pathogen yield (1) (CDC unpublished data, 2019), and the median interval from onset of limb weakness to CSF collection was short in 2016 (3 days) and again in 2018 ( 2 days). However, only 3\% of CSF specimens yielded a pathogen in 2018, similar to data from previous outbreak years. These data suggest that routine EV/RV PCR testing of CSF is unlikely to confirm the cause of these outbreaks $(1,8,14)$. This could represent the absence of viral shedding into the CSF or might reflect limitations in the timing of specimen collection from viremia, because CSF is collected only after onset of neurologic symptoms and not during the febrile or respiratory illness.

Although 44\% of confirmed AFM cases in 2018 had an enterovirus or rhinovirus identified in respiratory specimens, approximately half were negative. Timing of respiratory specimen collection improved in 2018 compared with that in 2016, but still occurred a median of approximately 3 days after the onset of limb weakness and 5 days after the onset of any respiratory illness. Shedding of viruses in the respiratory tract can be transient, so delays in specimen collection could contribute to negative findings. Because conventional testing has not been successful in identifying pathogens in the majority of AFM cases, testing strategies have been expanded to include approaches to identifying immune responses directed toward viruses implicated in AFM, such as measuring pathogen-specific antibody responses to infection. Timely specimen collection can optimize both conventional and novel testing approaches. 
Surveillance data from 2018 indicate that most patients received recommended medical care (13), with evidence of prompt hospitalization, and lumbar puncture and/or MRI shortly after the onset of limb weakness, indicating heightened clinical awareness and successful public health outreach since surveillance for AFM was implemented in 2014. Improved understanding of the different characteristics of confirmed and non-AFM cases, including a history of febrile or respiratory symptoms preceding onset of limb weakness and a predominance of upper limb involvement has helped to differentiate AFM from other forms of acute limb weakness (15).

AFM can progress rapidly and might require respiratory support (14-17); patients evaluated with signs and symptoms consistent with AFM should be hospitalized for close monitoring. In the absence of a confirmatory diagnostic test for AFM, management decisions for individual patients in the acute setting should be informed by careful review of the patient's signs and symptoms, laboratory testing, MRI results, and other test results, including electromyography, and in close consultation with infectious disease specialists and neurologists. To help with clinical management, the Acute Flaccid Myelitis Workgroup and the Transverse Myelitis Association (https://myelitis.org/ living-with-myelitis/resources/afm-physician-support-portal/) offer a 24-hour clinical consultation service with physicians at the University of Texas Southwestern's Transverse Myelitis Center or Johns Hopkins University Transverse Myelitis Center, established in 2019, for providers with questions about patients suspected to have AFM. Although studies on treatment have not been systematically evaluated for effectiveness, CDC, in collaboration with experts in multiple disciplines, developed interim considerations for the management of AFM patients (https://www.cdc.gov/acute-flaccid-myelitis/hcp/ clinical-management.html), which do not indicate a preference for or against any of the commonly employed treatments for AFM, including intravenous immunoglobulin, steroids, and plasmapheresis. Physical rehabilitation might also improve long-term outcomes if implemented during the acute phase of illness (14); thus, early recognition of AFM is important so that clinicians might implement targeted clinical management with the potential to improve patient outcomes.

Because AFM is uncommon, ensuring that all suspected cases are reported to public health is vital to collecting clinical information and specimens from each patient. There continues to be a notable delay in reporting suspected cases of AFM by clinicians to public health authorities. Delays might impede important provider outreach activities to increase awareness and the early collection of specimens for pathogen detection, particularly when there is an increase in cases.
To provide additional specificity for reporting of patients with suspected AFM to health departments, the Council of State and Territorial Epidemiologists modified the clinical criteria for reporting of patients suspected of AFM in June 2019 to include MRI evidence of spinal lesions with at least some gray matter involvement, in addition to acute flaccid limb weakness. ${ }^{\dagger}$ These changes to the case definition more clearly reflect the cumulative clinical and epidemiologic surveillance data collected for AFM over the past 5 years. It is important to note that the clinical diagnosis of AFM by a physician might differ from case definitions used for public health surveillance. Whereas an AFM diagnosis is based on a physician's comprehensive assessment of the affected patient, public health surveillance requires standardized criteria to evaluate overall morbidity, mortality, and seasonal trends and provide consistency in measurements from year to year.

The findings in this report are subject to at least three limitations. First, national AFM surveillance relies on passive reporting and clinician awareness, which can result in underreporting. Second, misclassification might occur since a confirmatory test for AFM is not available. Lack of submission of all requested specimen types from each patient suspected to have AFM limits the ability to fully characterize the laboratory profile of all AFM cases. Finally, although national data on long-term outcomes are not yet available, CDC and state and local health departments are investigating long-term outcomes of AFM patients and other risk factors that might affect the development of AFM.

Improving the understanding of AFM is a public health priority. The overall rarity of this condition and absence of a confirmatory test highlight the need for increased vigilance among providers seeing pediatric patients with acute onset of flaccid limb weakness in the late summer and fall. Ongoing national AFM surveillance will provide an important bridge between research and public health response and will be critical for the development of optimal treatment and prevention recommendations.

\rceil$^{\dagger}$ https://www.cste.org/page/PositionStatements. 


\section{Acknowledgments}

Vaccine Preventable Diseases Surveillance coordinators at local and state health departments; Carolyn Gould, Erin Staples, Amanda Panella, Emily McDonald, Marc Fischer, National Center for Emerging and Zoonotic Infectious Diseases Arbovirus Laboratory, CDC; AFM Task Force members; Sarah Hopkins, Children's Hospital of Philadelphia; Daniel Pastula, National Center for Emerging and Zoonotic Infectious Diseases, CDC; Catherine Otten, Seattle Children's Hospital; CDC AFM Outbreak Response Team.

Corresponding author: Adriana Lopez, alopez@cdc.gov, 404-639-8369.

${ }^{1}$ Division of Viral Diseases, National Center for Immunization and Respiratory Diseases, CDC.

All authors have completed and submitted the ICMJE form for disclosure of potential conflicts of interest. No potential conflicts of interest were disclosed.

\section{References}

1. Sejvar JJ, Lopez AS, Cortese MM, et al. Acute flaccid myelitis in the United States, August-December 2014: results of nationwide surveillance. Clin Infect Dis 2016;63:737-45. https://doi.org/10.1093/ $\mathrm{cid} /$ ciw372

2. Midgley CM, Watson JT, Nix WA, et al.; EV-D68 Working Group. Severe respiratory illness associated with a nationwide outbreak of enterovirus D68 in the USA (2014): a descriptive epidemiological investigation. Lancet Respir Med 2015;3:879-87. https://doi. org/10.1016/S2213-2600(15)00335-5

3. Sejvar JJ, Bode AV, Marfin AA, et al. West Nile virus-associated flaccid paralysis. Emerg Infect Dis 2005;11:1021-7. https://doi.org/10.3201/ eid1107.040991

4. Ooi MH, Wong SC, Clear D, et al. Adenovirus type 21-associated acute flaccid paralysis during an outbreak of hand-foot-and-mouth disease in Sarawak, Malaysia. Clin Infect Dis 2003;36:550-9. https://doi. org/10.1086/367648

5. Bitnun A, Yeh EA. Acute flaccid paralysis and enteroviral infections. Curr Infect Dis Rep 2018;20:34. https://doi.org/10.1007/ s11908-018-0641-x

6. Aliabadi N, Messacar K, Pastula DM, et al. Enterovirus D68 infection in children with acute flaccid myelitis, Colorado, USA, 2014. Emerg Infect Dis 2016;22:1387-94. https://doi.org/10.3201/eid2208.151949
7. Knoester M, Helfferich J, Poelman R, Van Leer-Buter C, Brouwer OF, Niesters HGM; 2016 EV-D68 AFM Working Group. Twenty-nine cases of enterovirus-d68-associated acute flaccid myelitis in Europe 2016: a case series and epidemiologic overview. Pediatr Infect Dis J 2019;38:16-21. https://doi.org/10.1097/INF.0000000000002188

8. Ruggieri V, Paz MI, Peretti MG, et al. Enterovirus D68 infection in a cluster of children with acute flaccid myelitis, Buenos Aires, Argentina, 2016. Eur J Paediatr Neurol 2017;21:884-90. https://doi.org/10.1016/j. ejpn.2017.07.008

9. McKay SL, Lee AD, Lopez AS, et al. Increase in acute flaccid myelitisUnited States, 2018. MMWR Morb Mortal Wkly Rep 2018;67:1273-5. https://doi.org/10.15585/mmwr.mm6745e1

10. Council of State and Territorial Epidemiologists. Revision to the standardized surveillance and case definition for acute flaccid myelitis. Atlanta, GA: Council of State and Territorial Epidemiologists; 2017. https://cdn.ymaws.com/www.cste.org/resource/ resmgr/2017PS/2017PSFinal/17-ID-01.pdf

11. Kilpatrick DR, Yang CF, Ching K, et al. Rapid group-, serotype-, and vaccine strain-specific identification of poliovirus isolates by real-time reverse transcription-PCR using degenerate primers and probes containing deoxyinosine residues. J Clin Microbiol 2009;47:1939-41. https://doi.org/10.1128/JCM.00702-09

12. Nix WA, Oberste MS, Pallansch MA. Sensitive, seminested PCR amplification of VP1 sequences for direct identification of all enterovirus serotypes from original clinical specimens. J Clin Microbiol 2006;44:2698-704. https://doi.org/10.1128/JCM.00542-06

13. Nix WA, Maher K, Johansson ES, et al. Detection of all known parechoviruses by real-time PCR. J Clin Microbiol 2008;46:2519-24. https://doi.org/10.1128/JCM.00277-08

14. Van Haren K, Ayscue P, Waubant E, et al. Acute flaccid myelitis of unknown etiology in California, 2012-2015. JAMA 2015;314:2663-71. https://doi.org/10.1001/jama.2015.17275

15. Hopkins SE. Acute flaccid myelitis: etiologic challenges, diagnostic and management considerations. Curr Treat Options Neurol 2017;19:48. https://doi.org/10.1007/s11940-017-0480-3

16. Nelson GR, Bonkowsky JL, Doll E, et al. Recognition and management of acute flaccid myelitis in children. Pediatr Neurol 2016;55:17-21. https://doi.org/10.1016/j.pediatrneurol.2015.10.007

17. Cramer N, Munjal N, Ware D, et al. New cluster of acute flaccid myelitis in Western Pennsylvania. Ann Emerg Med 2019;19:30051-4. https:// doi.org/10.1016/j.annemergmed.2019.01.024 